\title{
Contorted and ordinary body postures in the human brain
}

\author{
Emily S. Cross $\cdot$ Emilie C. Mackie $\cdot$ George Wolford • \\ Antonia F. de C. Hamilton
}

Received: 15 May 2009 / Accepted: 10 November 2009 / Published online: 27 November 2009

(C) The Author(s) 2009. This article is published with open access at Springerlink.com

\begin{abstract}
Social interaction and comprehension of nonverbal behaviour requires a representation of people's bodies. Research into the neural underpinnings of body representation implicates several brain regions including extrastriate and fusiform body areas (EBA and FBA), superior temporal sulcus (STS), inferior frontal gyrus (IFG) and inferior parietal lobule (IPL). The different roles played by these regions in parsing familiar and unfamiliar body postures remain unclear. We examined the responses of this body observation network to static images of ordinary and contorted postures by using a repetition suppression design in functional neuroimaging. Participants were scanned whilst observing static images of a contortionist or a group of objects in either ordinary or unusual configurations, presented from different viewpoints. Greater activity emerged in EBA and FBA when participants viewed contorted compared to ordinary body postures. Repeated presentation of the same posture from different viewpoints lead to suppressed responses in the fusiform gyrus as well as three regions that are characteristically activated by observing moving bodies, namely STS, IFG and IPL. These four regions did not distinguish the image viewpoint or the plausibility of the posture. Together, these data define a broad
\end{abstract}

\author{
E. S. Cross · A. F. de C. Hamilton \\ School of Psychology, University of Nottingham, \\ University Park, Nottingham NG7 2RD, UK \\ E. S. Cross ( $\square)$ \\ Department of Psychology, \\ Max Planck Institute for Human Cognitive and Brain Sciences, \\ Stephanstraße 1a, 04103 Leipzig, Germany \\ e-mail: cross@cbs.mpg.de \\ E. C. Mackie · G. Wolford \\ Center for Cognitive Neuroscience, \\ Dartmouth College, Hanover, NH 03755, USA
}

cortical network for processing static body postures, including regions classically associated with action observation.

Keywords Body representation - fMRI · EBA .

Contortion $\cdot$ Mirror neuron system

\section{Introduction}

As humans, we are inherently social creatures who spend a large portion of our time interacting with other individuals. The human body can convey a rich array of socially relevant information, including intention, emotion, and identity of any given individual. With growing interest in social cognition and how we understand our fellow humans, the question of how we represent other people's bodies becomes increasingly important (Peelen and Downing 2007). In the present paper, we examine the brain regions that represent ordinary and contorted static postures of the human body independent of viewing angle.

Previous studies have identified several brain regions that respond strongly when viewing the human body in different contexts. Within the temporal lobe, posterior superior temporal sulcus (pSTS), the middle temporal gyrus (MTG) and a portion of the fusiform gyrus respond robustly when viewing representations of the human body (Giese and Poggio 2003; Peelen and Downing 2005c; Peelen and Downing 2005a; Hodzic et al. 2009). Additionally, the inferior parietal lobule (IPL) is active when viewing human bodies (Grèzes and Decety 2001; Hodzic et al. 2009), as is the inferior frontal gyrus (IFG; Decety and Grèzes 1999; Buccino et al. 2001; Rizzolatti and Craighero 2004). However, the different roles performed by each of these regions in encoding static ordinary and extraordinary body postures are less clear. 
A posterior portion of the middle temporal gyrus, near the inferior temporal sulcus, responds more robustly to static representations of the human body compared to any other kind of stimuli tested, and has been termed the extrastriate body area (EBA; Peelen and Downing 2005c). This region responds selectively to still images of human bodies or body parts, stick figures and silhouettes of bodies (Downing et al. 2004; Downing et al. 2006; Peelen and Downing 2007), in preference to images of objects or human faces. Transcranial magnetic stimulation of EBA disrupts matching of body forms and not actions (Urgesi et al. 2007). Neuroimaging and neurostimulation studies report larger EBA responses to biomechanically impossible, compared to possible, static body images (Avikainen et al. 2003) or action sequences (Costantini et al. 2005). This evidence suggests that this portion of the MTG might be driven more by body-form information that is not necessarily related to action execution within an individual's normal movement repertoire.

A region with similar properties to $\mathrm{EBA}$ can be found within the mid-fusiform gyrus, on the ventral surface of the temporal lobes, and is termed the fusiform body area (FBA; Peelen and Downing 2005b). FBA is adjacent to, but anatomically and functionally distinct from, the more widely studied fusiform face area (Schwarzlose et al. 2005; Peelen et al. 2006), and responses within FBA tend to be rightlateralised when identifying human bodies (Hodzic et al. 2009). Few studies have attempted to parse the distinct contributions of EBA and FBA. However, it seems that EBA responds more strongly to representations of individual body parts or incomplete representations of the human body, whilst FBA responds more robustly to complete representations of the human body (Taylor et al. 2007).

The representation of static bodies in EBA and FBA contrasts with representations of the moving human body (such as videos of actions or real-life movements) in parietal, frontal and superior temporal regions. Neurons in superior temporal sulcus (STS) respond to both static (Grossman and Blake 2002; Giese and Poggio 2003) and moving postures (e.g. Grossman and Blake 2002; Cross et al. 2006). This region is commonly thought of as a biological motion-processing centre within the primate brain (Giese and Poggio 2003; Hein and Knight 2008). Many studies of action understanding localise IPL and IFG by contrasting dynamic to static action scenes (Keysers and Fadiga 2008; Del Giudice et al. 2009). The activation of IPL and IFG during both action observation and action execution (Grèzes and Decety 2001) has led to suggestions that these regions contain mirror neurons and encode other people's actions in relation to one's own actions (Rizzolatti and Craighero 2004). Evidence in favour of this role for IFG and IPL in dynamic, self-referential action representation can be found in studies of the role of action familiarity. IFG and IPL show stronger responses to familiar compared to novel actions (Calvo-Merino et al. 2005; Cross et al. 2006), which suggest that action embodiment is important in driving these brain regions. Candidi et al. (2008) have recently demonstrated that TMS to premotor cortex disrupts the perception of possible, but not impossible, actions, which again supports the role of action familiarity in driving these regions.

An influential model of body perception has proposed distinct representations of static body form and of body motion (Giese and Poggio 2003). This sophisticated hierarchical model has been mapped onto neuroanatomy, with the suggestion that certain regions within the occipitotemporal cortex represent static form (including EBA and FBA), whilst adjacent regions (including middle temporal cortex and kinetic occipital cortex) and more anterior regions (such as the premotor cortex) represent body motion. In this model, the STS has a role in both form and motion processing. This model is consistent with the research reviewed above and predicts a clear distinction between lateral and inferior temporal cortices, which encode static postures with stronger responses to impossible postures, whilst IPL, IFG and pSTS encode dynamic stimuli with a preference for familiar actions.

In the present study, we aimed to link past studies of familiarity of moving bodies and research on static body postures to obtain a fuller understanding of body representation in the brain. Further investigation is important because past studies are limited in several ways. First, many previous studies have used TMS to investigate body posture perception (e.g. Urgesi et al. 2007; Romani et al. 2005; Candidi et al. 2008). TMS is a powerful method for demonstrating that a brain region is necessary for a cognitive function, but is limited to examining only one or two brain regions in an experiment. The present study uses static stimuli in conjunction with functional imaging to examine body posture representations simultaneously within a broader set of areas of interest.

Second, the stimuli used to examine static body representations in past studies are typically shown from the same viewpoint, so matches between stimuli could be achieved using 'surface characteristics' of the image such as the shape of the body part outline. Limited behavioural studies have examined more abstract, viewpoint-independent representations of body postures (Reed and Farah 1995; Daems and Verfaillie 1999; Lawson et al. 2009). To date, there is increasing interest in the neural substrates of such viewpoint-independent representations (Pourtois et al. 2009), but such representations of the human body remain unexplored.

Third, the stimuli used in previous studies may not have been optimal for examining the representation of static body postures in IPL and IFG. Previous studies using static 
stimuli featured implied motion, so the role of the motor system in encoding static action stimuli without implied motion is less clear (e.g. Urgesi et al. 2007; Costantini et al. 2005; Stevens et al. 2000). Furthermore, the 'impossible postures' stimuli used in previous studies might be too extreme, or too aversive, to drive activity in IPL and IFG. Thus, in the present study, we use static images of contorted, but not humanly impossible postures.

The present paper aims to examine the representation of static ordinary and contorted postures in two novel ways. First, we test for brain regions with an overall preference for bodies compared to objects, and brain regions with a preference for possible postures or contorted postures. A previous study suggests that the region of the occipitotemporal cortex identified as EBA responds more robustly to bodies compared to objects (Peelen and Downing, 2005b). In terms of preference for contorted or ordinary configurations, there is evidence to suggest that EBA may prefer contorted postures (Avikainen et al. 2003; Costantini et al. 2005), whilst the IPL and IFG may prefer possible postures (Cross et al. 2006; Candidi et al. 2008). Second, we use the repetition suppression method to localise representations of posture, independent of viewpoint, and representations of viewpoint, independent of posture. Repeated viewing of the same posture should lead to a suppression of the BOLD response in brain regions, which encode that posture, even if the viewing angle changes (Grill-Spector and Malach 2001; Grill-Spector et al. 2006; Hamilton and Grafton 2006; Hamilton and Grafton 2008; Andresen et al. 2009). This method can therefore enable us to determine which brain regions contain viewpoint-independent postural representations.

\section{Materials and methods}

Eighteen healthy participants $(11$ female, mean age $=$ 21.3 years) gave informed consent to take part in this study. Seventeen were right-handed (Oldfield 1971), had normal or corrected-to-normal vision and were naïve with respect to the purpose of the experiment. The study was approved by the Committee for the Protection of Human Subjects at Dartmouth College and performed in accordance with the ethical standards set forth in the 1964 Declaration of Helsinki.

\section{Experimental design}

Stimuli were photographs of a person or a group of three objects that fell into one of two categories: ordinary and contorted (person) or ordinary and impossible (objects). Ordinary human photos showed a female contortionist in a meaningless, but easy-to-perform posture. Contorted human photos showed the same female in a contorted body posture that very few people (other than contortionists) are capable of performing. All participants were queried on completion of the experiment as to whether they could perform any of the contorted postures, and each participant responded unanimously that he or she could not perform any of the contorted postures. A mixture of standing and floor postures were used in each set of photos (Fig. 1, top two rows). Object configurations featured three common objects arranged together. For the impossible configurations, one of the objects was suspended in mid air with a clear fishing line, which was invisible in the resulting stimulus photographs (Fig. 1, bottom two rows). Photographs

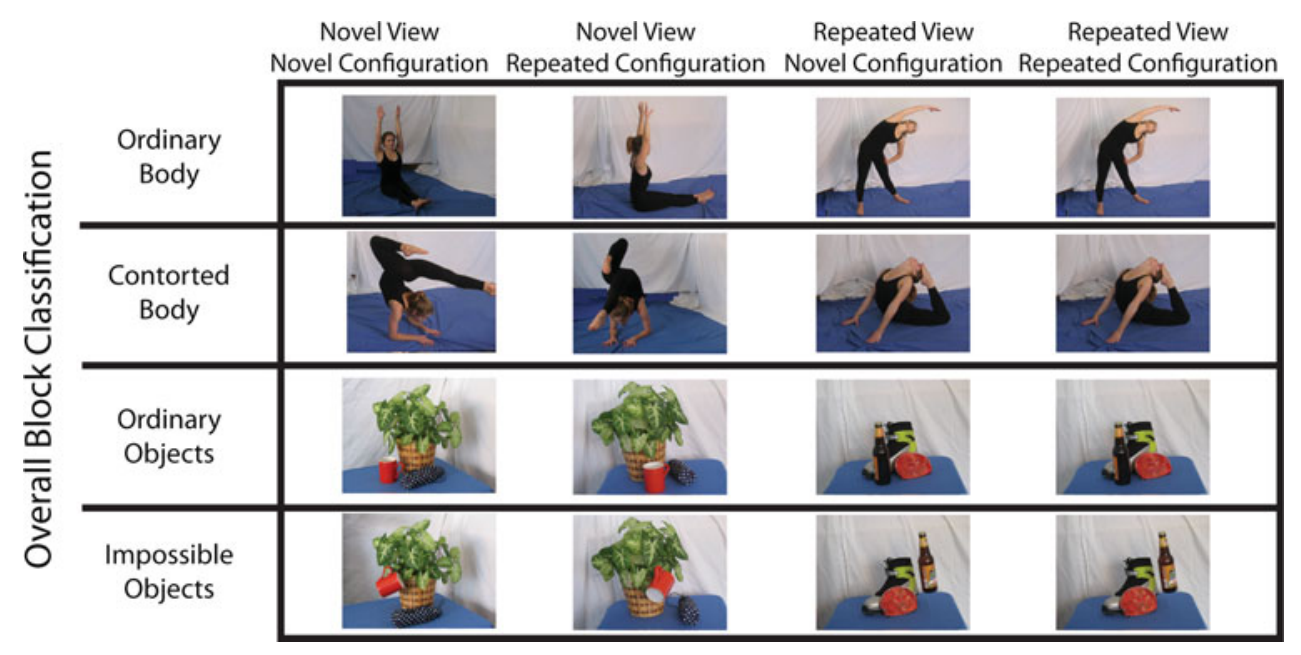

Fig. 1 Stimuli and experimental setup. The first and third rows illustrate examples of postures and object configurations from an experimental block that featured ordinary configurations. The second and fourth rows illustrate exemplar postures and objects from the contorted or impossible condition. For each experimental photograph, the view and posture could be different (novel) or the same (repeated).
Randomly interspersed throughout each block of trials was one trial in which two photographs were shown in rapid succession, in which the model's hand or foot changed angle, or in which one of the objects moved slightly in space. Participants' task was to watch for these trials and to press the response button whenever they detected apparent motion 
were taken with a Canon Powershot A620 and a Canon PowerShot A470 digital camera, each with 7.1 pixel resolution. Photographs were then resized to 400 by 300 pixel bitmap images with a horizontal/vertical resolution of $96 \mathrm{dpi}$ in Adobe Photoshop for presentation in the experiment.

During scanning, stimuli were presented in blocks, with ten pictures in each block appearing on the screen for $1.8 \mathrm{~s}$ each, with a $0.5 \mathrm{~s}$ blank separating each picture, for a total block duration of $23 \mathrm{~s}$. Each block contained only images from a single stimulus category (ordinary bodies, contorted bodies, ordinary objects, abnormal objects). Participants completed a total of three fMRI runs, each comprising 12 blocks of trials, with each block type presented three times in a pseudorandom order.

During all trials, participants were instructed to simply look at the images and perform a simple alerting task to make sure that they closely attended to photographs being presented. For the alerting task, participants made a key press when they saw either the human figure or one of the objects make an apparent movement. Apparent motion for these stimuli was produced by showing two photographs for $0.9 \mathrm{~s}$ each in succession with a change in the angle of one of the joints, which gave the impression of movement. As an example, a photograph of the model with a flexed foot might have immediately followed a pose where her foot was pointed, thus giving the illusion of a foot movement. An alerting trial lasted $1.8 \mathrm{~s}$ followed by a $0.5 \mathrm{~s}$ blank screen, just like normal trials. One alerting trial was presented in each block. Responses were not recorded, but were monitored throughout the experiment to ensure that participants were alert and completed the task. All stimuli were presented with Cogent running under Matlab 6.5, which permitted synchronisation with the scanner and precise timing of stimuli presentation.

To probe the neural representations of body postures and viewing angle in more detail, we embedded a repetition suppression (RS) design within this block design. For each postural or object configuration, two stimulus photographs were taken simultaneously from two cameras positioned approximately $90^{\circ}$ apart, giving two 'views' of that configuration. This meant that stimuli could be sequenced so that a trial showed a novel posture or a repeated posture (relative to the one trial before) and each posture could be shown from a novel viewpoint or a repeated viewpoint. This gave a $2 \times 2$ factorial design with factors posture and viewpoint, each with the levels novel and repeated (See columns of Fig. 1). Within each block, stimuli fell equally into the cells of this design and were pseudorandomly ordered to allow an event-related analysis of the BOLD signal time locked to each stimulus type. This manipulation of stimulus ordering within each block gives a hybrid block RS design, which gives great flexibility to data analysis.
Data collection and analysis

The experiment was carried out in a $3 \mathrm{~T}$ Philips Intera Achieva scanner using an eight-channel phased array coil and 30 slices per TR (3.5 mm thickness, $0.5 \mathrm{~mm}$ gap; TR, $1988 \mathrm{~ms}$; TE, $35 \mathrm{~ms}$; flip angle, 90; field of view, $24 \mathrm{~cm}$; matrix $80 \times 80$ ). For each of the three functional runs, the first two brain volumes were discarded, and the following 174 volumes were collected and stored.

To remove sources of noise and artefact, functional data were realigned, unwarped and normalised to the MNI template with a resolution of $3 \times 3 \times 3 \mathrm{~mm}$ in SPM2. Following this, $8 \mathrm{~mm}$ smoothing was applied to the images. A design matrix was fitted for each subject, with one regressor for each of the eight trial types illustrated in Fig. 1 plus one regressor for alerting trials. Nine similar regressors were fitted for the trials where participants saw objects in normal or unusual configurations, but those trials are not analysed here. All trials were modelled as events (zero duration) and convolved with the standard haemodynamic response function. Covariates of non-interest (a session mean, a linear trend and six movement parameters derived from realignment corrections) were included in the design. SPM2 was used to compute parameter estimates (beta) and contrast images (containing weighted parameter estimates) for each comparison at each voxel.

Two sets of contrasts were assessed at the second level in a random effects analysis.

1. First, we analysed the data as a block design, independent of repetition suppression effects. To determine which regions respond most robustly to human bodies compared to inanimate objects, we calculated the main effect of bodies (bodies $>$ objects). We report in this section only results that fell within the set of clusters showing stronger activation to bodies than objects. This ensures that we focus only on brain regions with a preferential response to the human body. Within a mask of the body $>$ objects contrast, we examined the main effect of postural plausibility. That is, we evaluated the contrast comparing contorted or impossible configurations to ordinary ones (contorted/impossible $>$ ordinary). We also tested the interaction of stimulus form (bodies-objects) with stimulus plausibility (contorted-ordinary).Finally, to identify brain regions that code the plausibility of body postures alone, we calculated the simple effect of implausible body postures (contorted $>$ ordinary) and the simple effect of plausible body postures (ordinary $>$ contorted) for all stimuli. These contrasts define which body-specific brain regions are more responsive when viewing postures that are contorted and beyond the physical limits of participants' own bodies and which regions are more 
responsive when viewing ordinary postures that are within the physical limits of participants' bodies.

2. The second set of imaging analyses evaluated repetition suppression effects for representations of posture, independent of viewpoint, and viewpoint, independent of posture. This analysis included trials showing both contorted and ordinary body postures, but not objects. Repetition suppression for posture was identified by calculating a contrast of novel posture $>$ repeated posture across both viewpoints and both ordinary and contorted body postures. Repetition suppression for viewpoint was calculated by contrasting novel $>$ repeated viewpoint across all postural configurations (novel, repeated, contorted and ordinary). We also tested for an interaction between these two contrasts. All repetition suppression contrasts were performed over the whole brain.
For all contrasts, we report regions that survive a threshold of $p<0.001$, uncorrected, and 10 voxels over the whole brain in Table 1. Parameter estimates were then extracted from peak voxels within these contrasts and plotted to illustrate the observed effects. To reduce false positives, we focus our discussion on results that fall within our predicted network of brain regions (EBA/FBA/STS/IPL/IFG).

\section{Results}

Block design analysis

\section{Bodies $>$ objects}

A stronger response to observing bodies compared to objects emerged in several cortical and subcortical regions

Table 1 Main effects of block design

\begin{tabular}{|c|c|c|c|c|c|c|c|c|}
\hline \multirow[t]{2}{*}{ Region } & \multirow[t]{2}{*}{ BA } & \multicolumn{3}{|c|}{ MNI coordinates } & \multirow{2}{*}{$\begin{array}{l}\text { Putative } \\
\text { functional name }\end{array}$} & \multirow{2}{*}{$\begin{array}{l}\text { Cluster } \\
\text { size }\end{array}$} & \multirow[t]{2}{*}{$t$ value } & \multirow[t]{2}{*}{$p_{\text {uncor. }}$ value } \\
\hline & & $x$ & $y$ & $z$ & & & & \\
\hline \multicolumn{9}{|l|}{ (a) Bodies $>$ objects } \\
\hline Left middle temporal gyrus* & 21 & -46 & -74 & 0 & EBA & 505 & 8.49 & $<0.0001^{\delta}$ \\
\hline Right middle temporal gyrus* & 21 & 48 & -82 & -2 & EBA & 906 & 8.10 & $<0.0001^{\delta}$ \\
\hline Left cerebellum & & -14 & -60 & -36 & & 11 & 5.81 & $<0.0001$ \\
\hline Right putamen & & 28 & 10 & -8 & & 26 & 5.75 & $<0.0001$ \\
\hline Right fusiform gyrus* & 37 & 44 & -60 & -26 & FBA & 44 & 5.64 & $<0.0001$ \\
\hline Left posterior cingulate cortex & 23 & -6 & -38 & 18 & & 180 & 4.90 & $<0.0001$ \\
\hline Left caudate nucleus & & -8 & -14 & -4 & & 14 & 4.72 & $<0.0001$ \\
\hline Left cerebellum & & -8 & -52 & -52 & & 11 & 4.01 & $<0.0001$ \\
\hline \multicolumn{9}{|l|}{ (b) Contorted > ordinary } \\
\hline Left middle temporal gyrus* & 37 & -48 & -64 & -6 & MTG & 764 & 8.58 & $<0.0001^{\delta}$ \\
\hline Right inferior temporal cortex* & 20 & 50 & -56 & -8 & ITG & 1071 & 6.88 & $<0.0001^{\delta}$ \\
\hline Right intraparietal sulcus & 7 & 22 & -52 & 44 & IPS & 39 & 4.77 & $<0.0001$ \\
\hline Left precuneus & 7 & -14 & -64 & 58 & & 46 & 4.55 & $<0.0001$ \\
\hline Left posterior fusiform gyrus & 37 & -38 & -50 & -26 & & 59 & 4.47 & $<0.0001$ \\
\hline Left parahippocampal gyrus & 36 & -14 & -44 & -14 & & 10 & 4.35 & $<0.0001$ \\
\hline Left hippocampus & & -34 & -12 & -32 & & 41 & 4.32 & $<0.0001$ \\
\hline Left superior parietal lobule & 7 & -30 & -40 & 48 & SPL & 51 & 4.25 & $<0.0001$ \\
\hline Anterior cingulate cortex & 24 & 0 & 16 & 12 & $\mathrm{ACC}$ & 17 & 4.23 & $<0.0001$ \\
\hline Right postcentral gyrus & 3 & 28 & -42 & 52 & $\mathrm{~S} 1$ & 15 & 4.21 & $<0.0001$ \\
\hline Right middle occipital gyrus & 18 & 44 & -86 & 4 & MOG & 23 & 4.07 & $<0.0001$ \\
\hline Right parahippocampal gyrus & 36 & 22 & -2 & -30 & & 12 & 3.98 & $<0.0001$ \\
\hline \multicolumn{9}{|l|}{ (c) Contorted > ordinary bodies only } \\
\hline Right inferior/mid temporal gyrus* & $20 / 21$ & 54 & -60 & -8 & EBA & 235 & 6.11 & $<0.0001^{\delta}$ \\
\hline Right fusiform gyrus* & 37 & 42 & -58 & -22 & FBA & 16 & 5.78 & $<0.0001$ \\
\hline Left middle temporal gyrus* & 21 & -48 & -68 & -2 & EBA & 134 & 5.56 & $<0.0001^{\delta}$ \\
\hline
\end{tabular}

MNI coordinates of peaks of relative activation within regions activated by main effect of bodies compared to objects, collapsed across configuration (a); main effect of contorted/impossible compared to ordinary configurations, collapsed across bodies and objects (b); and main effect of contorted compared to ordinary postural configurations, for bodies only, masked by the bodies $>$ objects contrast (c)

Asterisk $(*)$ denotes activations within our a priori network of interest, and a ${ }^{\delta}$ denotes activations significant at the cluster-corrected level of $p<0.05$ 
(Table 1a). The most robust activations were found in the bilateral occipitotemporal cortex, likely within the extrastriate body area (Fig. 2). A large cluster of activation was also found within the fusiform gyrus, likely in the fusiform body area. Several additional clusters from subcortical regions are included in the table for completeness, but as these do not fall within our network of predicted brain regions, we do not discuss them further.

\section{Ordinary and contorted/impossible configurations}

No brain regions responded more robustly to observing ordinary body and object configurations compared to contorted or impossible configurations. However, the inverse contrast revealed reliable activation across several occipital, temporal and parietal regions (Table $1 \mathrm{~b}$ ). The most robust activations were observed in the bilateral inferior temporal cortex. Other activations from within the predicted collection of regions emerged in the right intraparietal sulcus and left posterior fusiform gyrus.

\section{Contorted $>$ ordinary bodies, masked by bodies $>$ objects}

To explore whether body-selective brain regions are also sensitive to postural plausibility, we evaluated the contrast of contorted $>$ ordinary bodies only, masked by regions that emerged from the bodies $>$ objects contrast (Table 1a). This analysis revealed activation in just three brain regions: bilateral inferior temporal cortex, likely corresponding to extrastriate body area, and right fusiform gyrus, likely corresponding to fusiform body area (Table 1c; Fig. 2). Each of these clusters falls within $3-10 \mathrm{~mm}$ of previously reported EBA and FBA activations (Peelen and Downing 2005b; Peelen and Downing 2007; Hodzic et al. 2009).

\section{Interaction of configuration and form}

One region located at $x=46, y=-64$ and $z=-6$, at the edge of the EBA cluster identified in the bodies $>$ objects contrast, showed an interaction between configuration and form. This is plotted in Fig. 3. This region does not differentiate between contorted and ordinary bodies, but preferentially responds to impossible compared to ordinary object configurations. The inverse contrast did not reveal any activations within the bodies $>$ objects mask or within our broader network of interest.

\section{Repetition suppression effects}

\section{Viewpoint-independent representations of postural configuration}

An analysis of brain regions responsive to novel body configurations revealed activations in frontal, parietal and temporal action circuits (Table 2a). In particular, the inferior frontal gyrus, inferior parietal lobule, posterior superior temporal sulcus and an area of the fusiform gyrus close to the FBA functional region (Peelen and Downing 2005b), all within the right hemisphere, showed increased BOLD signal on presentation of a novel body posture compared to
Fig. 2 Brain regions showing a main effect of plausibility (contorted > ordinary; in red), a main effect of responding to bodies compared to objects (in green); and the simple effect of greater responses to contorted bodies compared to ordinary bodies (overlap of previous two contrasts; in yellow). Significant activity emerged in bilateral middle temporal gyrus, corresponding to the extrastriate body area (EBA), and right fusiform gyrus, corresponding to the fusiform body area (FBA) when viewing contorted postures compared to ordinary, non-contorted postures. Below the brain figure, parameter estimates (SPM betas) are plotted for these three regions for illustrative purposes only

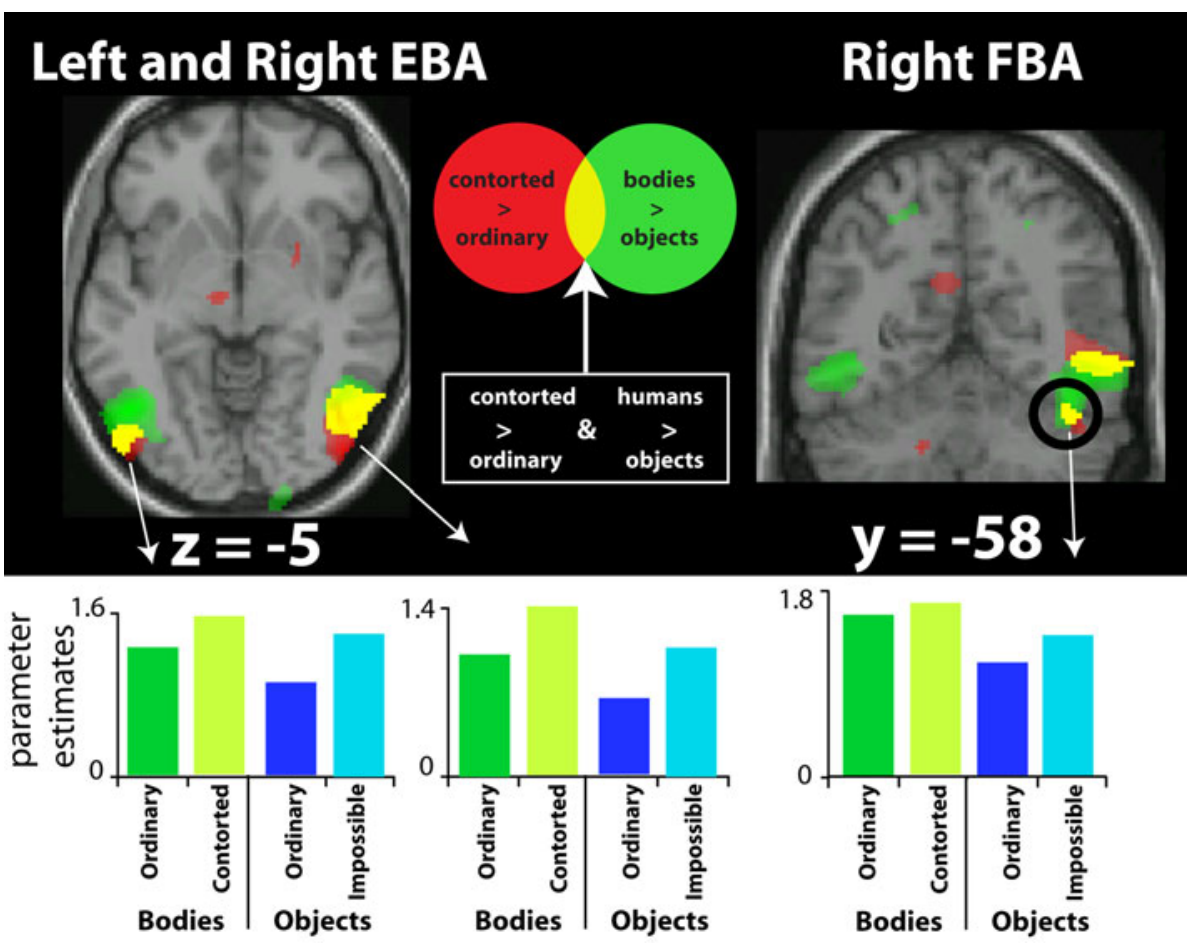




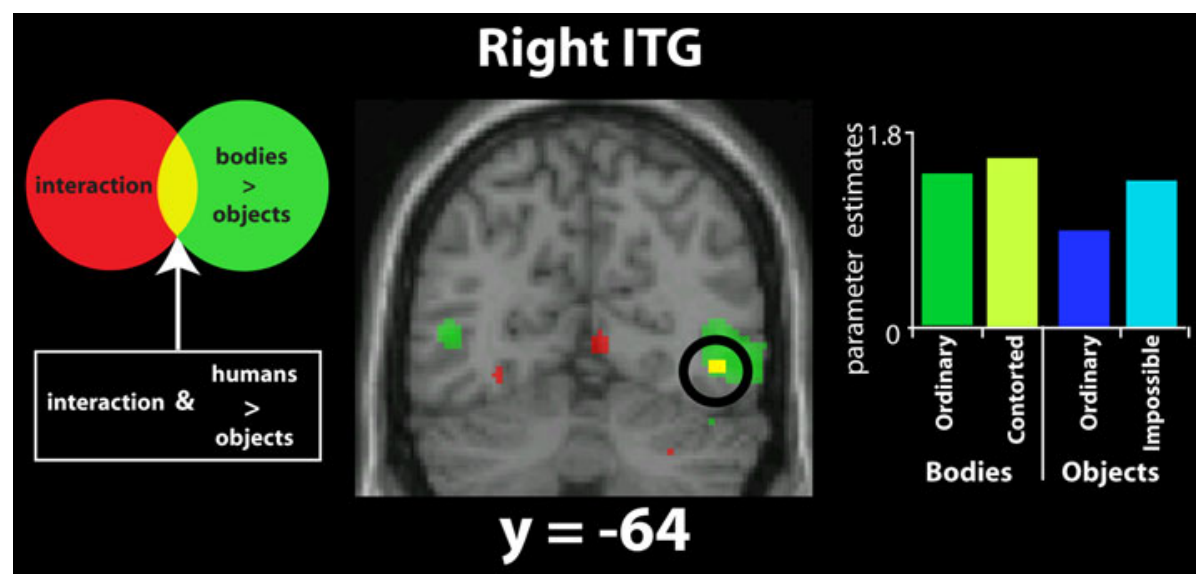

Fig. 3 Brain regions showing an interaction between configuration (contorted/impossible $>$ ordinary) and form (bodies $>$ objects) are illustrated in red. Regions from the interaction that fall within the bodies $>$ objects contrast (green) are represented by the overlap between these two contrasts, in yellow. One significant cluster of activation from the conjuction of these two contrasts emerged in a portion of the temporooccipital cortex, in the inferior temporal gyrus (ITG) within the EBA cluster. To the right of the brain figure are the parameter estimates (SPM betas) for this region, for illustrative purposes only

Table 2 Repetition suppression effects

\begin{tabular}{|c|c|c|c|c|c|c|c|}
\hline \multirow[t]{2}{*}{ Region } & \multirow[t]{2}{*}{ BA } & MNI coordinat & & \multirow{2}{*}{$\begin{array}{l}\text { Putative } \\
\text { functional name }\end{array}$} & \multirow{2}{*}{$\begin{array}{l}\text { Clust. } \\
\text { size }\end{array}$} & \multirow[t]{2}{*}{$t$ value } & \multirow[t]{2}{*}{$p_{\text {uncor. }}$ value } \\
\hline & & $y$ & $z$ & & & & \\
\hline
\end{tabular}

(a) RS posture, viewpoint independent

$\mathrm{R}$ inferior frontal gyrus*
$\mathrm{R}$ fusiform gyrus*
$\mathrm{R}$ inferior temporal gyrus
$\mathrm{L}$ middle frontal gyrus
$\mathrm{R}$ post. superior temp gyrus*
$\mathrm{R}$ postcentral gyrus
$\mathrm{R}$ supramarginal gyrus*

$\begin{array}{rrrrl}46 & 56 & 26 & 18 & \text { IFG } \\ 37 & 48 & -48 & -28 & \text { FBA } \\ 20 & 46 & 10 & -40 & \text { ITG } \\ 9 & -16 & 32 & 42 & \text { MFG } \\ 22 & 42 & -56 & 18 & \text { pSTS } \\ 3 & 44 & -22 & 34 & \text { SII } \\ 7 & 68 & -26 & 26 & \text { IPL }\end{array}$

(b) RS viewpoint, posture independent

$\begin{array}{lll}\text { L frontomedial wall } & 10 & -6 \\ \text { L orbitofrontal cortex } & 12 & -28 \\ \text { R medial prefrontal cortex } & 10\end{array}$

(c) Interaction 1: RS for posture and viewpoint

\begin{tabular}{|c|c|c|c|c|c|c|c|c|}
\hline $\mathrm{R}$ cingulate gyrus & 31 & 12 & -8 & 42 & & 236 & 6.18 & $<0.0001$ \\
\hline $\mathrm{R}$ cerebellum & & 2 & -42 & -54 & & 29 & 4.48 & $<0.0001$ \\
\hline $\mathrm{R}$ superior parietal lobule & 7 & 18 & -26 & 50 & SPL & 24 & 4.03 & $<0.0001$ \\
\hline \multicolumn{9}{|c|}{ d) Interaction 2: RS for posture and viewpoint } \\
\hline $\mathrm{R}$ pulvinar & & 16 & -28 & 0 & & 17 & 4.71 & $<0.0001$ \\
\hline $\mathrm{R}$ middle frontal gyrus & 46 & 18 & 48 & 10 & MFG & 14 & 4.25 & $<0.0001$ \\
\hline
\end{tabular}

MNI coordinates of peaks of relative activation within regions from the body-only contrasts demonstrating repetition suppression for novel, compared to repeated postural configurations, collapsed across contorted and ordinary postures, and novel and repeated viewpoints (a); repetition suppression for novel compared to repeated viewpoints, collapsed across contorted and ordinary postures, and novel and repeated postural configurations (b); and the interactions between repetition suppression for postural configuration and viewing angle (c and d)

Asterisk $(*)$ denotes activations within our a priori network of interest

a repeated posture. In each of these clusters, the robust response to a novel posture was suppressed when the same posture was repeated on a second trial, regardless of the viewing angle or the plausibility of the posture. This pattern of activations suggests that FBA, pSTS, IPL and IFG encode a body's postural configuration, independent of the viewing angle (Grill-Spector and Malach 2001; Hamilton and Grafton 2008). Figure 4 illustrates the foci of activation 
Fig. 4 Selection of brain regions showing $\mathrm{RS}$ for posture, independent of viewing angle. Significant suppression was seen for repeated postures compared to novel postures within the right fusiform gyrus, right posterior superior temporal suclus (pSTS), right inferior frontal gyrus (IFG) and right inferior parietal lobule (IPL). Parameter estimates (SPM betas) are plotted below the brain figures for each region, for illustrative purposes only ( $n$ novel, $r$ repeated, $V$ viewpoint)
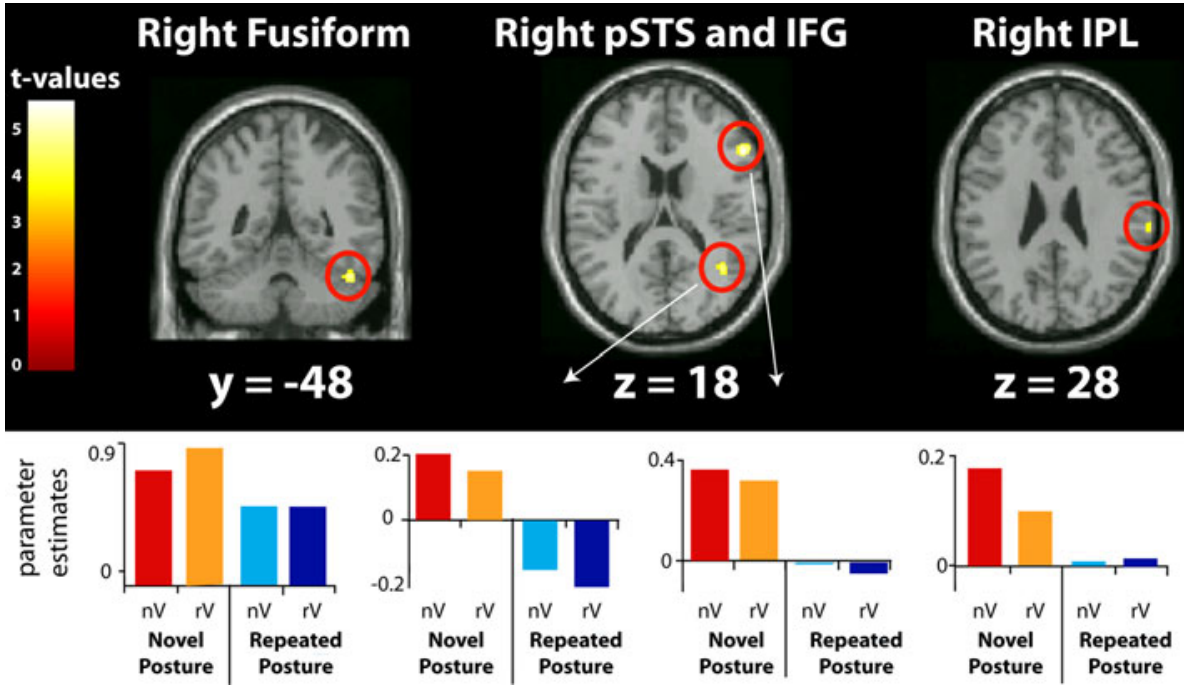

in these four regions along with their corresponding parameter estimates.

\section{Other RS contrasts}

The analysis of brain regions responsive to novel viewing angles did not reveal any activation within the predicted network of brain regions (Table 2b). This suggests that novel viewing angles of the human body are not sufficient to evoke responses in body-sensitive brain regions. We also tested for interactions in both directions between $\mathrm{RS}$ for posture and viewing angle. These analyses did not reveal any significant activation within the predicted network of brain regions (Table $2 \mathrm{c}, \mathrm{d}$ ).

\section{Discussion}

We found evidence of selective responses to different features of human postures within the hypothesised body representation network (lateral and ventral temporal lobes, IPL and IFG). In particular, regions likely to be EBA and FBA show a stronger response to contorted postures (compared to ordinary postures), whilst IFG, IPL, pSTS and an anterior portion of the fusiform gyrus are most responsive to novel postural configurations, independent of viewpoint. These results inform our understanding of how the brain processes static information from other people's bodies. The implications of each of these findings are considered in turn.

Representation of postural complexity: EBA and FBA

We observed two clusters of voxels in the inferior portion of the middle temporal gyrus and one cluster in the posterior, medial fusiform gyrus that are more strongly activated when viewing bodies in contorted postures that an observer cannot physically perform, compared to viewing ordinary postures. Importantly, these regions also responded more robustly to presentation of bodies compared to presentation of objects. This pattern of activation allows us to define these regions as EBA and FBA, which characteristically respond to bodies more than objects.

It is interesting to note that, in the parameter estimates illustrated in Fig. 2, EBA and FBA show stronger responses to impossible objects compared to possible objects, as well as the defining strong response to human bodies. That is, even when the stimuli come from the nonpreferred category (objects), these brain regions still show some distinction between ordinary and impossible configurations. This is consistent with recent findings that some regions of lateral occipital cortex do not show strict category selectivity, but show evidence of encoding faces, house or objects (Pourtois et al. 2009). Indeed, our analysis of the interaction between form and configuration found that a portion of EBA does not differentiate between different body configurations, but does differentiate ordinary from impossible object configurations (Fig. 3). This further supports the idea that some of these visual regions might not be as domain specific as suggested by earlier studies (e.g. Downing et al. 2006). As Pernet et al. (2007) argue, neuroimaging research on category specificity is yet to conclusively demonstrate brain activation findings consistent with true functional specialisation. The main effects and interaction from the present study clearly demonstrate the need for future work to precisely parse how extraordinary arrangements of body parts and objects are coded within adjacent regions of lateral and ventral temporal cortices.

It is useful to consider the similarities between our data and previous studies of EBA. Greater BOLD responses have been recorded in EBA when viewing impossible postures or actions (e.g. Avikainen et al. 2003; Costantini et al. 
2005; Downing et al. 2006; Candidi et al. 2008). Our findings extend these previous results by showing that an observed posture does not have to be physically impossible for the human body to perform, per se, to reliably activate EBA. Rather, the critical factor appears to be whether the perceived posture is impossible for the observer to perform. Such an interpretation is consistent with the notion that a key function of EBA might be to extract body-form cues that are either unrelated, impossible or beyond what the viewer's body can do (Avikainen et al. 2003; Downing et al. 2006; Candidi et al. 2008).

However, there are also differences between the functional profile of the activations we report and those described in a prior study. Downing et al. (2006) showed that EBA responded more robustly to incoherent compared to coherent action sequences. They suggested that EBA activity was suppressed by the presentation of similar postures, as found in the coherent action sequences. In contrast, we did not find any evidence of RS for postures in EBA or any MTG regions. This could indicate that our clusters are not EBA, or could reflect the viewpoint manipulation we used. Downing et al. did not alter the viewpoint, and so the reduced BOLD response they report for coherent action sequences might reflect viewpoint-dependent RS in EBA. The idea that posture representations in EBA might be viewpoint dependent is consistent with a model of biological motion processing (Giese and Poggio 2003) in which EBA contains 'snapshot' neurons that selectively respond to visually presented human bodies from specific viewpoints. This interpretation is also consistent with other neuroimaging studies on EBA's selectivity to viewpoint (Chan et al. 2004; Saxe et al. 2006; Pourtois et al. 2009).

Viewpoint-independent encoding of postures: fusiform gyrus, pSTS, IFG and IPL

Four regions of the body representation network showed the same pattern of BOLD responses to viewing body postures. Fusiform gyrus, pSTS, IFG and IPL all showed a reduced signal when the same body posture was repeated, independent of viewpoint. Activation of these areas was not modulated by the plausibility of the posture, and these regions did not show an overall preference for bodies compared to objects. This pattern of data can best be interpreted in terms of the population coding model of RS, in which repeated presentation of a certain stimulus feature causes reduced activity in the neuronal population that encodes that stimulus feature, leading to a suppression of the BOLD signal. A release from suppression is indicative of engagement of a different neural population within the same brain region (Grill-Spector et al. 2006; Hamilton and Grafton 2006; Krekelberg et al. 2006; Grafton and Hamilton 2007). Thus, our data imply that fusiform gyrus, pSTS, IFG and
IPL all contain populations of neurons that encode static human body postures independent of viewpoint. The implications of this interpretation vary for the different regions.

The fusiform gyrus cluster showing RS for posture did not overlap with the fusiform cluster showing main effects of bodies and of contorted postures in the block design contrasts. Thus, we cannot conclusively attribute this fusiform cluster to FBA. Nevertheless, our group activation in the fusiform gyrus is within 3-5 mm from FBA peaks reported previously (Peelen and Downing 2005b). It is thus possible that our fusiform activation includes at least some of the region that has been functionally localised as FBA. The identification of distinct peaks within FBA that are sensitive to contorted bodies and to repeated body postures is a novel finding. This suggests that FBA might have distinct nodes for coding postural complexity and postural configuration, independent of viewpoint, a notion consistent with recent work on FBA by Schwarzlose et al. (2008).

Based on previous work from our laboratory with observation of skilled and unskilled whole-body actions (Cross et al. 2006; Cross et al. 2009a, b), we predicted that the putative 'mirror neuron system' regions of IFG and IPL would prefer ordinary (familiar) postures compared to contorted postures, which are rarely or never performed. Our results did not support this prediction. Instead, we found clusters within IFG and IPL that showed RS for both ordinary and contorted body postures. This could be because different neural populations within the same brain areas code for novelty of body configurations and highly skilled action embodiment. Alternatively, we may have been premature in claiming that IPL and IFG respond specifically to familiar actions. Other studies have demonstrated comparable responses in these regions during observation of biomechanically possible and impossible action sequences (Costantini et al. 2005; Romani et al. 2005). This may reflect generalisation across different kinds of action and is consistent with a model of action understanding proposed by Schubotz (2007), which may help facilitate observational learning (e.g. Cross et al. 2009b).

A critical distinction between the current study and many previous studies of STS/IPL/IFG (Decety and Grèzes 1999; Grèzes et al. 2001; Calvo-Merino et al. 2005; MolnarSzakacs et al. 2006; Cross et al. 2009a) is that we used still images of a model in static postures, whereas previous studies used movies of moving actors or still images of actors in the middle of a dynamic action. Because we do not directly compare brain activations to static and dynamic stimuli within one study, it is not possible to draw strong conclusions about the relative activation of the body representation network by static and dynamic stimuli. We did not systematically evaluate participants' perceptions of implied motion for the static body posture photographs used in the present study, but every posture was stable and 
was designed to appear static. It remains possible that implied motion in our postures is a factor in driving the activation of STS and IPL/IFG, but future work will be needed to investigate this.

Our data show that the fusiform gyrus, STS, IPL and IFG contain abstract, view-invariant representations of static postures. This finding is novel and exciting for several reasons. First, it confirms the notion that IPL and IFG are not only responsive to moving displays of actions, but they also respond to static postures. Whilst several past studies have also suggested that the inferior frontal and parietal components of the mirror neuron system might respond to static postures (Urgesi et al. 2006; Candidi et al. 2008), these prior investigations have only looked at static representations of implied actions. Here, we show that static postures that were not designed to strongly imply action (unlike those of Urgesi et al. (2006) and Candidi et al. (2008)) are sufficient to find reliable RS in the right IFG and IPL. Second, our results may present a challenge to the model Giese and Poggio (2003) in which all body representations are viewpoint dependent. Instead, it implies that fusiform gyrus, STS, IPL and IFG contribute to a network that provides a three-dimensional, viewpoint-independent body posture representation.

Third, our results may also shed light on an earlier behavioural study that provided some of the first evidences for interaction between perceptual and motor processes (Reed and Farah 1995). Reed and Farah showed typical participants' images of bodies in complex postures from two different viewpoints and asked them to judge if the postures were the same or different. Performance was worse when participants concurrently moved their own limbs, and this effect was specific to the observed and acting limb. For example, if the two figures shown in the task differed only in arm posture, and the participant concurrently moved his arm, reaction times were slower than if the participant concurrently moved his leg. This study provided some initial evidence that we use a representation of our own body in making judgements about other people's bodies. However, if static bodies were represented only in EBA/FBA, whilst motor planning involves mainly IPL/IFG, it would be difficult to account for Reed and Farah's data at a brain level. Our experiment demonstrates that IPL and IFG are involved in the viewpoint-independent representation of the human body, and one possibility is that the interference observed by Reed and Farah (1995) occurs in these brain regions. This provides a key link between behavioural research and our understanding of body representation in the human brain.

\section{Conclusions}

Taken together, the present results suggest that the representation of ordinary and contorted body postures is spread across several brain regions spanning much of the cerebral cortex. In line with prior studies on impossible postures and action sequences (Avikainen et al. 2003; Costantini et al. 2005; Downing et al. 2006), we found that left and right EBA respond more robustly to contorted body postures, compared with ordinary postures. In contrast, distinct portions of the fusiform gyrus show preferential responses to contorted postures and viewpoint-independent encoding of body postures. This finding is consistent with prior evidence for this region responding robustly to intact bodies (Peelen and Downing 2005b; Taylor et al. 2007), and provides a useful point of departure for further investigation into FBA's specific contributions to body processing. The superior temporal sulcus, inferior frontal gyrus and inferior parietal lobule also demonstrated repetition suppression for viewpoint-independent encoding of body configurations. This suggests that neurons within these regions respond less to whether a particular static pose can be achieved by the observing individual, and more to novel limb configurations. Future experiments will be needed to determine the relationship between static body posture representations and dynamic, experience-dependent representations within these brain regions. Such work should further illuminate the differential contributions that EBA, FBA, IPL and IFG make to representations of the human body.

Acknowledgments We thank RX Ramsey, Barbara Vogt and the two anonymous reviewers for helpful comments on earlier versions of this manuscript. This work was funded by a National Institutes of Health National Research Service Award (F31-NS056720) to ESC.

Open Access This article is distributed under the terms of the Creative Commons Attribution Noncommercial License which permits any noncommercial use, distribution, and reproduction in any medium, provided the original author(s) and source are credited.

\section{References}

Andresen DR, Vinberg J, Grill-Spector K (2009) The representation of object viewpoint in human visual cortex. Neuroimage 45:522-536

Avikainen S, Liuhanen S, Schurmann M, Hari R (2003) Enhanced extrastriate activation during observation of distorted finger postures. J Cogn Neurosci 15:658-663

Buccino G, Binkofski F, Fink GR, Fadiga L, Fogassi L, Gallese V, Seitz RJ, Zilles K, Rizzolatti G, Freund HJ (2001) Action observation activates premotor and parietal areas in a somatotopic manner: an fMRI study. Eur J Neurosci 13:400-404

Calvo-Merino B, Glaser DE, Grèzes J, Passingham RE, Haggard P (2005) Action observation and acquired motor skills: an FMRI study with expert dancers. Cereb Cortex 15:1243-1249

Candidi M, Urgesi C, Ionta S, Aglioti SM (2008) Virtual lesion of ventral premotor cortex impairs visual perception of biomechanically possible but not impossible actions. Soc Neurosci 3:388-400

Chan AW, Peelen MV, Downing PE (2004) The effect of viewpoint on body representation in the extrastriate body area. Neuroreport $15: 2407-2410$ 
Costantini M, Galati G, Ferretti A, Caulo M, Tartaro A, Romani GL, Aglioti SM (2005) Neural systems underlying observation of humanly impossible movements: an FMRI study. Cereb Cortex 15:1761-1767

Cross ES, Hamilton AF, Grafton ST (2006) Building a motor simulation de novo: observation of dance by dancers. Neuroimage 31:1257-1267

Cross ES, Hamilton AF, Kraemer DJ, Kelley WM, Grafton ST (2009a) Dissociable substrates for body motion and physical experience in the human action observation network. Eur J Neurosci 30:1383-1392

Cross ES, Kraemer DJ, Hamilton AF, Kelley WM, Grafton ST (2009b) Sensitivity of the action observation network to physical and observational learning. Cereb Cortex 19:315-326

Daems A, Verfaillie K (1999) Viewpoint-dependent priming effects in the perception of human actions and body postures. Vis Cogn 6:665-693

Decety J, Grèzes J (1999) Neural mechanisms subserving the perception of human actions. Trends Cogn Sci 3:172-178

Del Giudice M, Manera V, Keysers C (2009) Programmed to learn? The ontogeny of mirror neurons. Dev Sci 12:350-363

Downing PE, Bray D, Rogers J, Childs C (2004) Bodies capture attention when nothing is expected. Cognition 93:B27-B38

Downing PE, Peelen MV, Wiggett AJ, Tew BD (2006) The role of the extrastriate body area in action perception. Soc Neurosci $1: 52-62$

Giese MA, Poggio T (2003) Neural mechanisms for the recognition of biological movements. Nat Rev Neurosci 4:179-192

Grafton ST, Hamilton AF (2007) Evidence for a distributed hierarchy of action representation in the brain. Hum Mov Sci 26:590-616

Grèzes J, Decety J (2001) Functional anatomy of execution, mental simulation, observation, and verb generation of actions: a metaanalysis. Hum Brain Mapp 12:1-19

Grèzes J, Fonlupt P, Bertenthal B, Delon-Martin C, Segebarth C, Decety J (2001) Does perception of biological motion rely on specific brain regions? Neuroimage 13:775-785

Grill-Spector K, Malach R (2001) fMR-adaptation: a tool for studying the functional properties of human cortical neurons. Acta Psychol (Amst) 107:293-321

Grill-Spector K, Henson R, Martin A (2006) Repetition and the brain: neural models of stimulus-specific effects. Trends Cogn Sci 10:14-23

Grossman ED, Blake R (2002) Brain areas active during visual perception of biological motion. Neuron 35:1167-1175

Hamilton AF, Grafton ST (2006) Goal representation in human anterior intraparietal sulcus. J Neurosci 26:1133-1137

Hamilton AF, Grafton ST (2008) Action outcomes are represented in human inferior frontoparietal cortex. Cereb Cortex 18:1160-1168

Hein G, Knight RT (2008) Superior Temporal Sulcus-It's My Area: Or Is It? J Cogn Neurosci

Hodzic A, Kaas A, Muckli L, Stirn A, Singer W (2009) Distinct cortical networks for the detection and identification of human body. Neuroimage 45:1264-1271

Keysers C, Fadiga L (2008) The mirror neuron system: new frontiers. Soc Neurosci 3:193-198

Krekelberg B, Boynton GM, van Wezel RJ (2006) Adaptation: from single cells to BOLD signals. Trends Neurosci 29:250-256
Lawson RP, Clifford CW, Calder AJ (2009) About turn: the visual representation of human body orientation revealed by adaptation. Psychol Sci 20:363-371

Molnar-Szakacs I, Kaplan J, Greenfield PM, Iacoboni M (2006) Observing complex action sequences: the role of the fronto-parietal mirror neuron system. Neuroimage 33:923-935

Oldfield RC (1971) The assessment and analysis of handedness: The Edinburgh Inventory. Neuropsychologia 9:97-113

Peelen MV, Downing PE (2005a) Is the extrastriate body area involved in motor actions? Nat Neurosci 8:125; author reply 125-126

Peelen MV, Downing PE (2005b) Selectivity for the human body in the fusiform gyrus. J Neurophysiol 93:603-608

Peelen MV, Downing PE (2005c) Within-subject reproducibility of category-specific visual activation with functional MRI. Hum Brain Mapp 25:402-408

Peelen MV, Downing PE (2007) The neural basis of visual body perception. Nat Rev Neurosci 8:636-648

Peelen MV, Wiggett AJ, Downing PE (2006) Patterns of fMRI activity dissociate overlapping functional brain areas that respond to biological motion. Neuron 49:815-822

Pernet C, Schyns PG, Demonet JF (2007) Specific, selective or preferential: comments on category specificity in neuroimaging. Neuroimage 25:991-997

Pourtois G, Schwartz S, Spiridon M, Martuzzi R, Vuilleumier P (2009) Object representations for multiple visual categories overlap in lateral occipital and medial fusiform cortex. Cereb Cortex 19:1806-1819

Reed CL, Farah MJ (1995) The psychological reality of the body schema: a test with normal participants. J Exp Psychol Hum Percept Perform 21:334-343

Rizzolatti G, Craighero L (2004) The mirror-neuron system. Annu Rev Neurosci 27:169-192

Romani M, Cesari P, Urgesi C, Facchini S, Aglioti SM (2005) Motor facilitation of the human cortico-spinal system during observation of bio-mechanically impossible movements. Neuroimage 26:755-763

Saxe R, Jamal N, Powell L (2006) My body or yours? The effect of visual perspective on cortical body representations. Cereb Cortex 16:178-182

Schubotz RI (2007) Prediction of external events with our motor system: towards a new framework. Trends Cogn Sci 11:211-218

Schwarzlose RF, Baker CI, Kanwisher N (2005) Separate face and body selectivity on the fusiform gyrus. J Neurosci 25:11055-11059

Schwarzlose RF, Swisher JD, Dang S, Kanwisher N (2008) The distribution of category and location information across object-selective regions in human visual cortex. Proc Natl Acad Sci USA 105:4447-4452

Stevens JA, Fonlupt P, Shiffrar M, Decety J (2000) New aspects of motion perception: selective neural encoding of apparent human movements. Neuroreport 11:109-115

Taylor JC, Wiggett AJ, Downing PE (2007) Functional MRI analysis of body and body part representations in the extrastriate and fusiform body areas. J Neurophysiol 98:1626-1633

Urgesi C, Moro V, Candidi M, Aglioti SM (2006) Mapping implied body actions in the human motor system. J Neurosci 26:7942-7949

Urgesi C, Candidi M, Ionta S, Aglioti SM (2007) Representation of body identity and body actions in extrastriate body area and ventral premotor cortex. Nat Neurosci 10:30-31 